\title{
Retinal Pigment Epithelium Tears: Risk Factors, Mechanism and Therapeutic Monitoring
}

\author{
Christoph R. Clemens Nicole Eter \\ Department of Ophthalmology, University of Münster Medical Centre, Münster, Germany
}

\section{Key Words}

Age-related macular degeneration - Retinal pigment epithelium · Retinal pigment epithelial detachment . Retinal pigment epithelium tear · Intravitreal injection . Spectral-domain optical coherence tomography

\begin{abstract}
Tears of the retinal pigment epithelium (RPE) are most commonly associated with vascularised RPE detachment due to age-related macular degeneration (AMD), and they usually involve a deleterious loss in visual acuity. Recent studies suggest an increase in RPE tear incidences since the introduction of anti-vascular endothelial growth factor (anti-VEGF) therapies as well as a temporal association between the tear event and the intravitreal injection. As the number of AMD patients and the number of administered anti-VEGF injections increase, both the challenge of RPE tear prevention and the treatment after RPE tear formation have become more important. At the same time, the evolution of retinal imaging has significantly contributed to a better understanding of RPE tear development in recent years. This review summarises the current knowledge on RPE tear development, predictive factors, and treatment strategies before and after RPE tear formation.
\end{abstract}

(c) 2015 S. Karger AG, Basel

\section{Introduction}

The introduction of anti-vascular endothelial growth factor (anti-VEGF) agents in the treatment of patients suffering from exudative age-related macular degeneration (AMD) has doubtlessly been a breakthrough in ophthalmology and has so far prevented severe vision loss in a great number of patients. Retinal pigment epithelium (RPE) tears represent a rare complication in the treatment of patients with exudative AMD and frequently result in a devastating loss of visual acuity [1-3].

RPE tears are known to occur as a natural result in the course of a retinal pigment epithelial detachment (PED) because of underlying choroidal neovascularisation (CNV), retinal angiomatous proliferation, or polypoidal choroidal vasculopathy. Since the beginning of intravitreal anti-VEGF therapies in patients with PED due to exudative AMD, RPE tears have increasingly been reported as a complication after injection.

As the number of injections has distinctly increased, clinicians more frequently face the question of RPE tear prevention or treatment after tear formation. The evolution of retinal imaging has significantly contributed to a better understanding of RPE tear development and proved to be a valuable tool in adapting therapy regimens in PED patients.

\section{KARGER}

E-Mail karger@karger.com

www.karger.com/oph
(C) 2015 S. Karger AG, Basel

0030-3755/15/2351-0001\$39.50/0
Christoph R. Clemens, MD

Department of Ophthalmology, University of Münster Medical Centre Domagkstrasse 15

DE-48149 Münster (Germany)

E-Mail Christoph.Clemens@ukmuenster.de 
This review highlights the current knowledge on RPE tear development, predictive factors indicating an impending RPE tear, as well as treatment strategies before and after RPE tear formation.

\section{Epidemiology}

RPE tears were first reported in 1981 by Hoskin et al. [4] as a complication in patients with PED due to AMD. RPE tears can be either part of the natural course of PED due to occult $\mathrm{CNV}$, retinal angiomatous proliferation, or polypoidal choroidal vasculopathy or they can occur in association with various treatments for neovascular AMD such as photodynamic therapy, laser photocoagulation, or transpupillary thermotherapy [5-7].

The incidence is reported to be between 5 and $27 \%$ in the elderly [1-21]. Casswell et al. [1] reported a spontaneous tear rate of vascular PEDs of $10 \%$. In today's clinical routine, most RPE tears seem to be closely associated with anti-VEGF treatments and have been reported for the substances pegaptanib, bevacizumab, ranibizumab, and aflibercept [8-11]. The first description of an RPE tear after intravitreal bevacizumab injection for the treatment of an occult CNV due to AMD was reported by Meyer et al. [12] in 2006.

Different RPE tear incidences are reported under different treatment agents and various treatment regimens [13$22]$. A retrospective study of 1,280 eyes treated with bevacizumab included 125 eyes with a vascularised PED (vPED) showing an RPE tear rate of $16.8 \%$ [13]. In a case series of 6 patients with vPED, Chang et al. [14] reported a tear incidence of $27 \%$ under a treatment with pegaptanib. Clemens et al. [15] reported a tear incidence of $25 \%$ in a prospective study of a monthly ranibizumab regimen in 40 patients with vPED. Recently, Sarraf et al. [16] presented results from a prospective randomised study on 0.5 or $2.0 \mathrm{mg}$ ranibizumab therapy based on a monthly or pro re nata regimen in eyes with vPED that showed an incidence of $14 \%$ (5/37 patients). Interestingly, 4 out of the 5 reported RPE tears occurred in the high-dosage group. Apart from a few case reports, there are no prospective data available yet on the RPE tear rate in vPED patients under a treatment with aflibercept [11]. A single case report exists on RPE tear development in the untreated fellow eye following antiVEGF therapy for treatment of neovascular AMD. A causative relation remains speculative [23].

Reported incidences of RPE tears in the literature must be interpreted cautiously as underlying patient collectives, treatment regimens, as well as the nomenclature of lesion morphology tend to be very heterogeneous. Notwithstanding, the higher incidences of RPE tears in eyes under anti-VEGF therapy in contrast to the natural history studies on PED eyes suggest an enhancing effect of anti-VEGF agents on RPE tear development. Comparative prospective studies will presumably not be carried out in the near future due to ethical issues.

\section{Imaging}

\section{Colour Fundus Photography}

Using funduscopy, an RPE tear may be suspected based on the presence of subretinal and sub-RPE blood, an often well-defined hyperpigmented line at the site of the rolled RPE, and a depigmented area corresponding to the exposed choroid [4]. Retinal or subretinal haemorrhages are clearly distinguishable from RPE tear areas in colour fundus photography (CFP) compared to other modalities. Haemorrhages may interfere with an RPE tear area detection in fundus autofluorescence (FAF) (fig. 1a, b), which makes CFP indispensable in this entity.

Sarraf et al. [24] introduced an RPE tear grading system including CFP. Grade 1 tears were defined as $<200$ $\mu \mathrm{m}$. Grade 2 tears were between $200 \mu \mathrm{m}$ and 1 disk diameter. Grade 3 tears were $>1$ disk diameter. Grade 4 tears were defined as grade 3 tears that involved the centre of the fovea.

\section{Fundus Autofluorescence}

In FAF imaging, areas of RPE tears exhibit a markedly reduced autofluorescent signal because RPE cells with fluorescent lipofuscin are lost [25]. Small RPE tears are more evident in FAF and are easier to detect than in CFP. The high contrast of these hypoautofluorescent areas, compared with the intact retina, allows for an easy and accurate determination of lesion boundaries (fig. 1b). The configuration of RPE tears is classified as unilobular if RPE areas are homogeneously formed and as multilobular if RPE defects consist of two or more lobes separated by a piece of intact RPE strand [26]. Over time, edges of hypoautofluorescence become hazier and less demarcated in FAF imaging. However, the very first morphologic changes seen in FAF at the RPE tear edges, described as hyperreflective dots of various diameters, are not seen until 3 months after the acute event of RPE tear formation. Remodelling processes at the border zone do not seem to be detectable by FAF imaging during the first few months [27]. In contrast to geographic atrophy in AMD, hypofluorescent areas in FAF due to RPE tear formation do not 

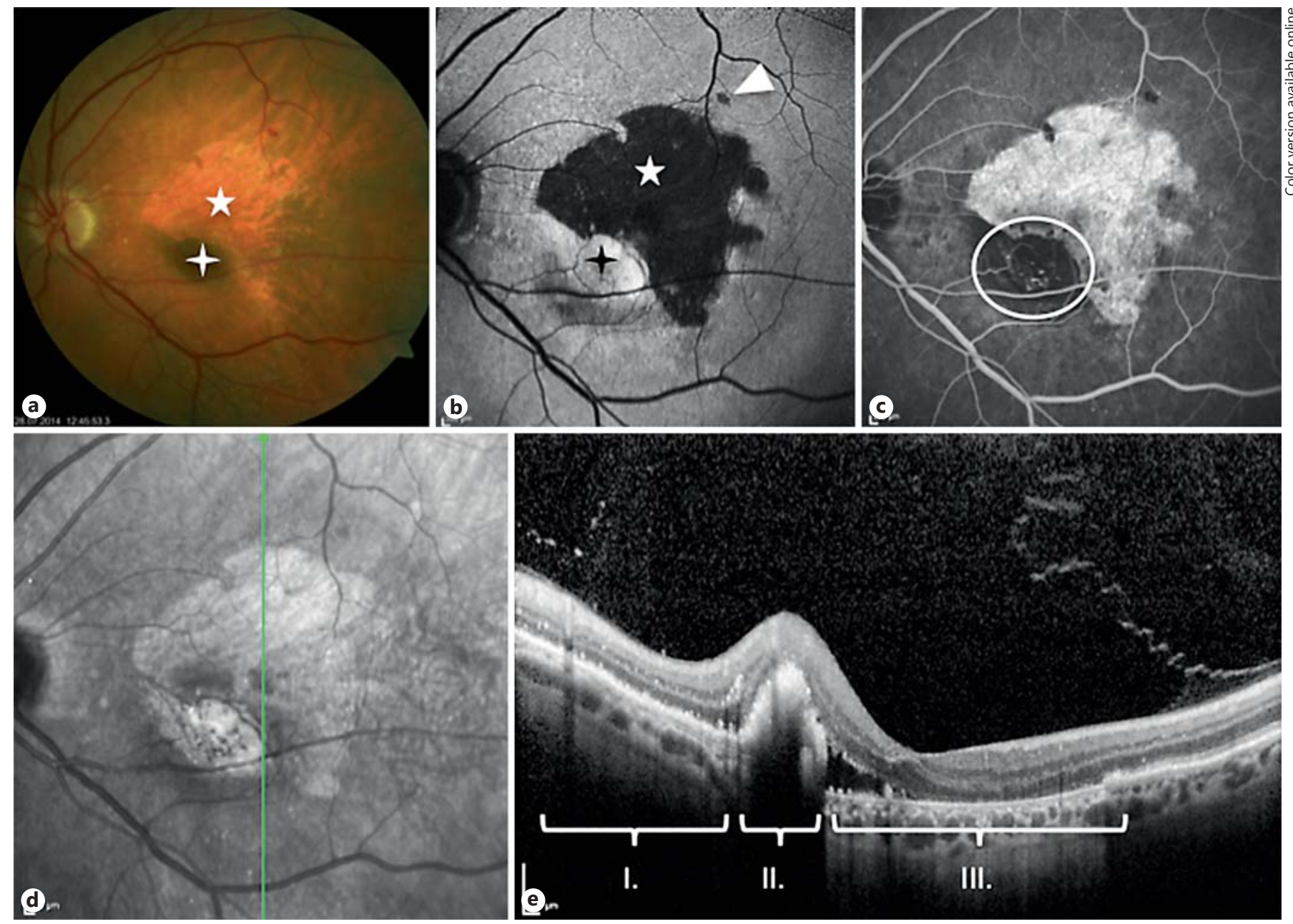

Fig. 1. Multimodal imaging of an exemplary patient with vPED due to AMD after RPE tear development. a CFP shows a depigmented area corresponding to the exposed choroid (star) and a hyperpigmented area corresponding to the enrolled RPE (diamond). Note the small retinal haemorrhage superiorly located to the RPE tear which is not clearly distinguishable from the hypoautofluorescent RPE tear area in the FAF modality as shown in $\mathbf{b}$ (arrowhead). b The cSLO FAF image shows a large, sharply delineated, hypoautofluorescent signal in the RPE tear area (star) as well

as a hyperautofluorescent area corresponding to the enrolled RPE (diamond). c Late-phase FLA reveals CNV in the inferior part of the lesion (circle) and a hyperfluorescent signal in the tear area. Notably, the RPE tear has occurred at the opposite side of the CNV localisation. d, e Combined cSLO near-infrared image (d) and SDOCT scan (e) showing a normal architecture of the outer retinal bands in the first part. In the second part, the free edge of torn RPE is being retracted towards the CNV. Note the missing RPE band in the RPE tear area representing the third part.

show patterns of abnormally increased FAF in the junctional zone of atrophy [28].

\section{Fluorescence Angiography}

Fluorescence angiography (FLA) demonstrates blocked fluorescence at the site of the rolled RPE and marked hyperfluorescence at the site of the exposed choroid (fig. 1c) [24]. In 2010, Sarraf et al. [24] described a thin defect at the margin of the PED with a subtle ring sign of hyperfluorescence in FLA. This observation was

found in RPE tears of grade 1 (greatest linear diameter $<200 \mu \mathrm{m}$ ) and represents a microscopic RPE defect detectable in corresponding spectral-domain optical coherence tomography (SD-OCT) scans. Lesions $>200 \mu \mathrm{m}$ present angiographically as small oval hyperfluorescent defects at the edge of the PED with early transmission and late staining and a subtle inner edge of hypofluorescent blockage. Larger RPE tears show a large crescentic area of early transmission hyperfluorescence with an adjacent patch of hypofluorescent blockage. 


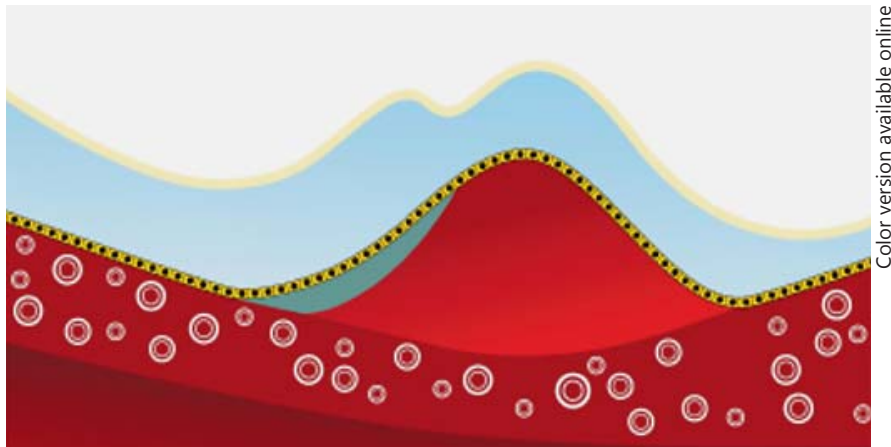

a

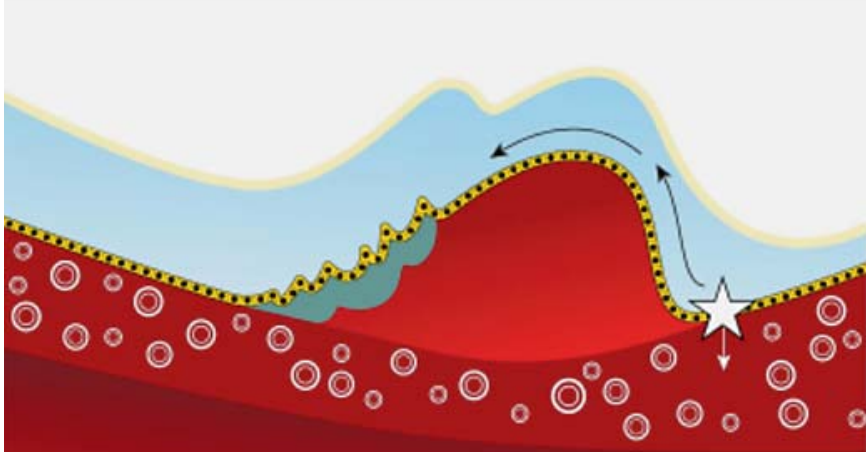

b

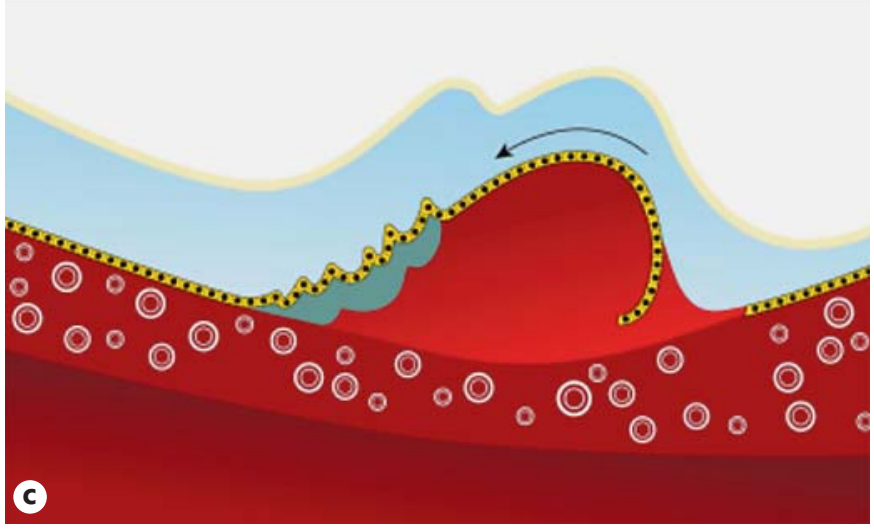

Fig. 2. Schematic drawing illustrating the proposed RPE tear mechanism in eyes with neovascular AMD after treatment with intravitreal anti-VEGF therapy. a The vPED contains the occult CNV membrane (blue-gray) adherent to the undersurface of the RPE monolayer (yellow-black cell layer). b Contraction of the $\mathrm{CNV}$ induces tensile forces that act in the plane of the RPE, applying the highest mechanical stress at the junction of the attached and detached RPE (star). At this very location, two opposite forces are acting: traction forces from CNV contraction and adhesive forces from the RPE that is still attached (arrows). c The increase in contraction eventually results in the anatomic failure of the RPE at the junction of attached and detached RPE. The free edge of torn $\mathrm{RPE}$ is being retracted towards the CNV. For colours, see online version.

\section{Spectral-Domain Optical Coherence Tomography}

In a classic RPE tear, three parts of the site can be distinguished on SD-OCT scans. In the first part, the outer retinal bands show a normal architecture. In the second part, the retina forms a dome-shaped detachment from the choroid. In this elevated zone, SD-OCT shows the free edge of a wavy, contracted RPE being retracted towards the CNV. The rolled RPE causes a hyperreflectivity and an intense back-shadowing, which completely masks the choroid. In the third part, the RPE is completely absent, and the neurosensory retina appears thinned. SD-OCT demonstrates an increased depth signal due to the absent RPE monolayer (fig. 1d). In some cases, an exudative reaction between the neurosensory retina and the choroid can be visualised in the follow-up after the acute event. In other cases, the neurosensory retina remains directly on the choroid [29].

\section{Mechanism of RPE Tear Formation}

The development of RPE tears may occur as a spontaneous process or following thermal laser, photodynamic therapy, or anti-VEGF therapy [2, 5, 8-11]. For many years, numerous authors have postulated an RPE tear mechanism based on the contraction of fibrovascular membranes [30-33]. Later on in the anti-VEGF era, an increase in the RPE tear incidence in AMD patients was interpreted as a confirmation of the established theory of tractional forces causing the tear event. Contraction of CNV membranes induces shrinkage of the RPE, which may cause an increased tension on the surface of the cavity. During this process, two opposite forces are acting on the marginal RPE: traction forces from CNV contraction and adhesive forces from the RPE that is still attached. The increase in contraction eventually results in the anatomic failure of the RPE at the junction of attached and detached RPE (fig. 2) [29, 34].

Multimodal imaging provides mechanistic insights into RPE tear development based on pre-tear and posttear architectural configuration of vPED. Spaide [35] assumed that the collection of material beneath the RPE represents a CNV within the PED. Contracture of this CNV adherent to the undersurface of the RPE applies the maximum traction at the junction of the attached and detached RPE representing the 'locus minoris resistentiae' and lying perpendicular to the CNV's contraction force. The contracted RPE monolayer comes to rest on the side of the $\mathrm{CNV}$, the origin of contraction, 
which becomes evident in SD-OCT or as hyperautofluorescence in FAF. Previous studies reported a tendency of RPE tears to develop at the temporal margin of the vPED lesion $[36,37]$. The reason for this site of predilection for RPE tear development remains unknown.

RPE tear formation in fibrovascular PED, in which the lesion cavity is entirely filled by the CNV membrane, is less frequent, presumably as contraction forces in response to an anti-VEGF therapy may spread evenly over the entire PED lesion exposing the RPE monolayer to less mechanical stress. In contrast, in serous-vascularised PED, the PED cavity is only partly filled by the CNV membrane [34]. Interestingly, RPE tears in patients under anti-VEGF therapy tend to occur within 1-3 months of the beginning of intravitreal treatment, whereas lateonset RPE tears tend to occur in treatment-naïve patients or in patients treated with photodynamic therapy [17].

In contrast to a conventional RPE tear, Ie et al. [38] hypothesised that the mechanism leading to a microrip of the RPE is based on hydrostatic forces alone producing a break at the margin of the PED. A microrip should not be regarded as a pre-stage RPE tear or the beginning of an RPE tear. Much more likely, microrips and RPE tears represent two separate entities based on different etiologic mechanisms as postulated by Ie et al. [38] in contrast to Sarraf et al. [39].

\section{Predicting Factors}

As an RPE tear usually results in a devastating loss of visual acuity, the identification of reliable risk factors is of great clinical importance. So far, several prognostic markers of an impending RPE tear have been described such as the PED lesion's height and diameter, hyperreflective lines in near-infrared images, a small ratio of CNV size to PED size, subretinal clefts, microrips, and duration of PED.

PED height has been reported as a predictor of RPE tears by Chan et al. [13]. They reported an increased prevalence of RPE tears in PED lesions higher than 400 $\mu \mathrm{m}$. Several other authors have addressed the issue of at what cut-off point PED height becomes a risk factor. Doguizi and Ozdek [19] statistically determined that 580 $\mu \mathrm{m}$ may be regarded as a cut-off point of PED height for the risk of an RPE tear. Similarly, Sarraf et al. [39] described a height of $550 \mu \mathrm{m}$ as a high-risk factor for the subsequent development of an RPE tear, and, addition- ally, Leitritz et al. [40] described an increasing probability of RPE tears particularly beyond the height of $400 \mu \mathrm{m}$. Moreover, Chiang et al. [20] hypothesised that an increased surface area as well as a large linear diameter of a subfoveal PED represent predisposing factors for RPE tear development (fig. 3a). Chan et al. [37] reported a stronger tendency towards tear development in those PED lesions that show a smaller ratio of CNV size to PED size (fig. 3b).

Detection of increased reflectance signals on confocal scanning laser ophthalmoscopy (cSLO) near-infrared images represents another predictive marker for an impending RPE tear (fig. 3c). These hyperreflective lines, which characteristically originate from the edge of PED lesions and usually spread like a funnel across the lesion, correspond to the CNV localisation observed in indocyanine green angiography. Additionally, hyperreflective lines in near-infrared images correlate with folds in the RPE as detectable in SD-OCT [34]. These findings indicate the mechanical stress caused by both CNV contraction and $\mathrm{CNV}$ relaxation.

Microrips of the RPE were firstly described as a leak at the edge of the RPE detachment with passage of fluorescein into the subretinal space [38]. Later on, Sarraf et al. [24] introduced a classification system of RPE tears and defined grade 1 as a thin defect at the margin of the PED with a subtle ring sign of hyperfluorescence in angiography as well as a microscopic RPE defect detectable in OCT. Clemens et al. [41] recently postulated to regard microrips as an RPE tear risk factor presenting a patient with microrips that developed a foveal RPE tear after one anti-VEGF injection. Microrips supposedly lower the threshold of RPE resistance, and an increase in contraction after anti-VEGF therapy may eventually result in the anatomic failure of the RPE (fig. 3d).

Mukai et al. [42] recently described the formation of a subretinal cleft prior to RPE tear development in 3 patients, suggesting this SD-OCT characteristic as a potential risk factor (fig. 3e). This subretinal cleft may be a result of increasing mechanic pressure in the sub-RPE space.

Finally, Doguizi and Ozdek [19] reported an inverse relationship between the duration of PED and RPE tear formation. In their study, the PED had been present for a distinctly shorter period in the RPE tear patient group. They postulated that a short duration of PED means that the neovascular process is fresh, with immature vessels. Because the immature vessels are more susceptible to anti-VEGFs, the response to anti-VEGF therapy may be more dramatic. 


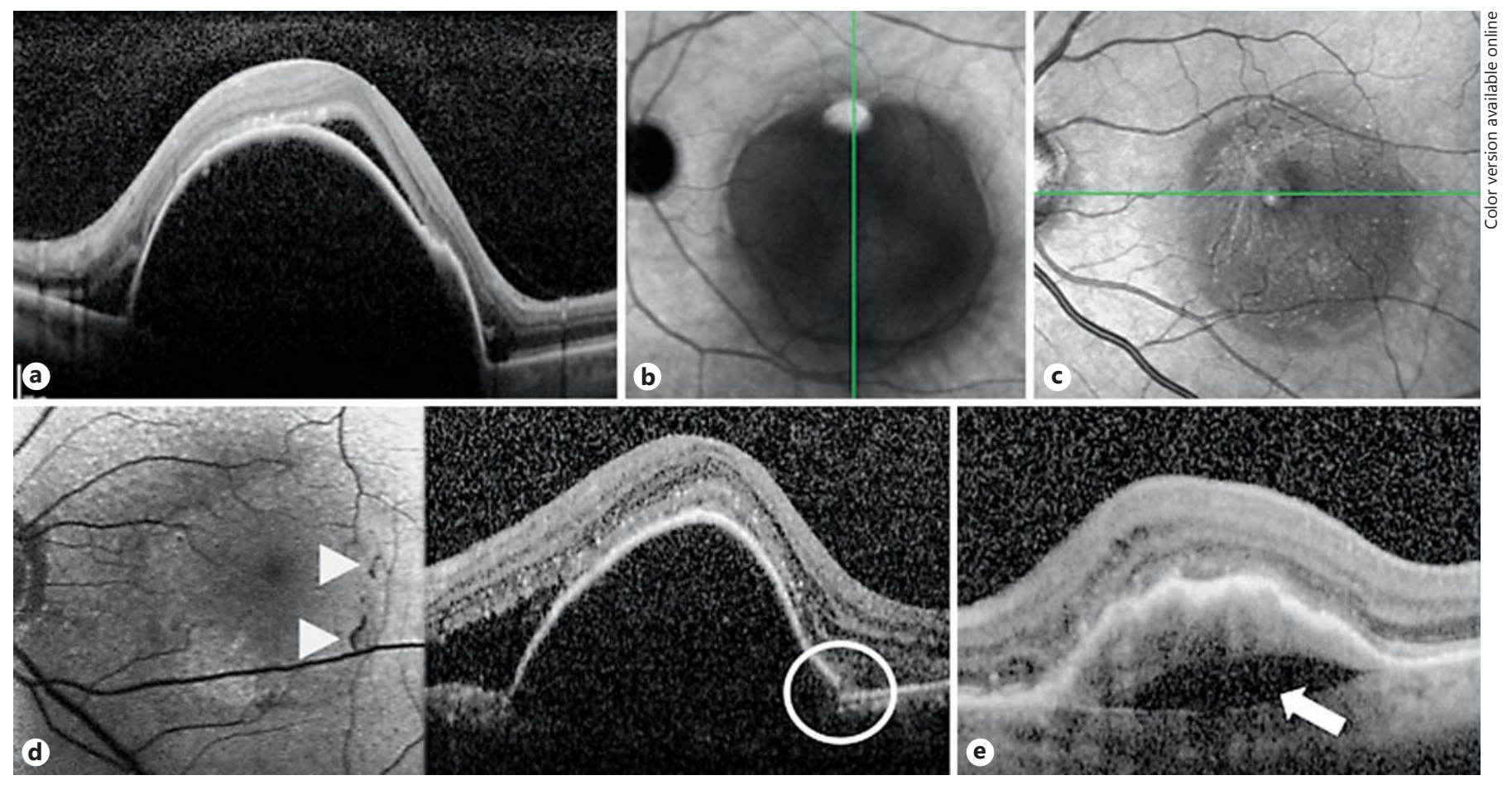

Fig. 3. RPE tear risk factors in patients with vPED due to AMD. a SD-OCT scan of a vPED lesion with both a large diameter and a large height. b Indocyanine green angiography image illustrating the concept of a small CNV/PED ratio. Hyperfluorescence superiorly represents a small CNV, while the round hypofluorescence represents the large dimensions of the $\mathrm{vPED}$ resulting in a very small ratio. c The cSLO near-infrared image of another vPED lesion shows hyperreflective lines originating from the edge of the
vPED that spread like a funnel across the lesion. d Combined cSLO FAF and SD-OCT depicting small hypoautofluorescences at the edge of a vPED indicating areas of microrips (arrowheads) that correspond to microscopic RPE defects (circle) detectable in the SD-OCT scan to the right. e Enhanced-depth imaging SD-OCT scan showing a subretinal cleft. The arrow points to a hyporeflective space underneath the sub-RPE neovascular tissue.

\section{Therapy in High-Risk Patients}

Clinicians are encouraged to look for established RPE tear risk factors prior to anti-VEGF therapy in patients with vPED due to AMD. A high-risk vPED patient is defined as showing one or more of the described RPE tear risk factors at the beginning or during the course of an antiVEGF treatment. In such high-risk patients, it is recommended to perform a thorough examination including SDOCT and FAF after each injection. If several risk factors accumulate or single risk factors significantly grow during an anti-VEGF treatment, we suggest to discontinue the injection therapy, to re-evaluate the PED lesion 1-2 weeks later, and to re-inject if signs of CNV contraction have declined, for instance, if RPE folds decline or hyperreflective lines disappear. Such an adapted regimen may make antiVEGF therapy safer with regard to RPE tear development in vPED patients at high risk. On the other hand, postponing therapy carries the risk of $\mathrm{CNV}$ progression.
Chan et al. [43] recently reported results from a prospective study comparing the outcomes of 0.5 versus $2.0 \mathrm{mg}$ intravitreal ranibizumab injections for treating vPED. The higher dose yielded more rapid reductions and more complete resolution of the PED; however, the incidence of RPE tear was higher in this group. The data suggest that the quantum of contraction forces on the PED lesion caused by the anti-VEGF agent must be carefully thought of. An overdosage may easily result in tear development, particularly in high-risk patients.

Most of the available data on RPE tear development after therapy exists on bevacizumab and ranibizumab, while only single case reports are available on aflibercept so far [11]. The receptor sequences of aflibercept provide a powerful VEGF binding (140 times that of ranibizumab), and the molecule has a 1-month intravitreal binding activity that exceeds both ranibizumab and bevacizumab. Unlike both anti-VEGF agents, aflibercept binds not only all isomers of the VEGF-A family but also VEGF-B 
and placental growth factor [44]. The clinical significance of these pharmacological differences is still unknown. However, one could postulate to use certain drugs for certain types of PED. Assuming a higher potency of CNV contraction of aflibercept, it may be reasonable to prefer ranibizumab in high-risk vPED patients and to use aflibercept in low-risk fibrovascular PED patients. One case report described an RPE tear development after two aflibercept injections in a vPED patient that had been treated with ranibizumab before [45]. However, at present, there are no further data available addressing this issue. Currently, a study group is recruiting patients to prospectively investigate the effect of aflibercept in vPED patients regarding the incidence of RPE tear development. Besides the issue of the most appropriate anti-VEGF agents for high-risk patients another unanswered question is whether a certain treatment regimen may be beneficial to adhere to regarding tear development in this patient group. At all events, particularly in high-risk PED eyes, patients must be informed about the risk of tear development during anti-VEGF treatment.

\section{Therapy after Tear Formation}

Controversy exists among clinicians regarding the treatment criteria after the formation of RPE tears. A retrospective analysis of data from three phase III randomised, multicentre clinical trials of ranibizumab for the treatment of neovascular AMD showed that, among patients who had an RPE tear, those treated with ranibizumab tended to have better visual acuity improvements [46]. Doguizi and Ozdek [19] found stable visual acuity results in 28 patients with continued anti-VEGF therapy after the formation of an RPE tear within a mean followup period of 20.6 months. The mean number of antiVEGF injections was 4.1 during this follow-up period. Final visual acuity was worse for larger RPE tears (grades 3 and 4) when compared with smaller tears (grades 1 and 2 ), which is in accordance with findings by Sarraf et al. [24]. Similar functional results were reported by Coco et al. [47]. Improvements in visual acuity have also been reported in patients with spontaneous RPE tears who were subsequently treated with anti-VEGF therapy [48]. Recently, Bartels et al. [49] reported a case of significant functional improvement in microperimetry and morphology after continuation of anti-VEGF therapy over 3 years because of persistent subretinal and intraretinal fluid after tear formation.
At present, there is no prospective clinical study available; however, an interventional multicentre trial is currently recruiting patients and investigates the effect of fixed monthly intravitreal injections of ranibizumab on visual acuity and retinal morphology over a study period of 12 months in 30 patients with an RPE tear due to neovascular AMD (ClinicalTrials.gov identifier: NCT01914159). Currently, most retina experts recommend continuing antiVEGF treatment after RPE tear development based on the presence of disease activity such as intra- or subretinal fluid and given that a functional benefit or stabilisation is expectable.

Nevertheless, re-injection after RPE tear has to be carefully evaluated as the RPE tear area may increase significantly under anti-VEGF therapy. Asao et al. [50] evaluated the effects of additional anti-VEGF therapy in 10 eyes with an RPE tear after anti-VEGF therapy over 12 months. They observed an increase in RPE tear area size $>20 \%$ in half of the included eyes. Clemens et al. [26] reported 3 patients initially presenting with a multilobular RPE tear, revealing a significant enlargement of the RPE tear with foveal involvement after another anti-VEGF injection. On the other hand, the same study showed that unilobular RPE tear defects remained stable during a 6-month follow-up period. Presumably, RPE strands between RPE tear lobes seem to prevent tear progression in multilobular RPE tears. Measuring RPE tear areas in these patients may have therapeutic consequences. If the RPE tear area in multilobular lesions increases, intravitreal anti-VEGF therapy should be postponed, and re-treatment should only be considered if signs of disease activity further increase. Additionally, in patients with small RPE tear areas outside the fovea and inactive CNV lesions, close followups are more reasonable than prompt re-injection.

Mukai et al. [51] observed two types of repair processes after RPE tear development in a small imaging study of 10 patients. Firstly, persistent subretinal fluid after the tear event seems to lead to subsequent repair with a thickened proliferative tissue at the area where the RPE was lost. Secondly, if an early and complete resolution of subretinal fluid after RPE tear development is achieved, the outer retina appears to be directly attached to Bruch's membrane without an ingrowth of proliferative tissue along Bruch's membrane. These observations suggest a beneficial effect on the macular morphology of a continuative anti-VEGF therapy after RPE tear development. Yet, whether the two repair mechanisms result in diverging functional results remains to be shown.

Mendis and Loris [52] presented a longitudinal FAF study in 14 RPE tear patients and reported an RPE resur- 
facing of the area debrided of RPE in 10 eyes and a progression of RPE cell loss in 4 eyes. In the former group, the recovery of the FAF signal occurred centripetally, from the edge of the area of the defect towards the centre, with the exception of the border where the rolled RPE was present.

Previous studies found that the RPE basement membrane is more conducive for RPE resurfacing, serving as a scaffolding structure, than the deeper collagen layer of Bruch's membrane, suggesting that the depth of the cleavage plane may have an impact on the extent of RPE resurfacing. Experimental studies showed that RPE cells repopulate from the edge to the centre rather than from residual cells remaining in the debrided area [53, 54].

Caramoy et al. [55] analysed RPE tissue remodelling in RPE tears based on FAF and SD-OCT imaging and observed evidence of resurfacing in small RPE tears, whereas RPE migration and proliferation may not occur in the right plane in large tears. Transplantation of the RPE using human embryonic stem cells and induced pluripotent stem cell-derived RPE is being developed and evaluated as a cell replacement therapy for AMD. Further studies are needed to assess its potential to restore some of the lost RPE function in patients suffering from an RPE tear [56].

\section{Conclusions}

Due to a growing number of intravitreal anti-VEGF injections in AMD patients, the incidence of RPE tear formation has increased. Thus, clinicians must be aware of strategies to prevent RPE tear development during antiVEGF therapy and to treat patients after RPE tear formation. An adapted treatment regimen in high-risk vPED may prevent patients from RPE tear development. After RPE tear formation, anti-VEGF treatment is recommended as long as activity signs are present. Multimodal imaging during anti-VEGF therapy in high-risk patients represents the key tool to detect risk factors and to make antiangiogenic treatment safer. Future studies must address several issues, such as which anti-VEGF agent is most appropriate in high-risk vPED patients, which treatment regimen is most beneficial after RPE tear development, and in how far can retinal imaging be improved to visualise RPE tear risk factors as early as possible and to identify new characteristics indicating an impending RPE tear.

\section{Disclosure Statement}

C.R. Clemens is associated with Heidelberg Engineering, Novartis, and Bayer; N. Eter is associated with Heidelberg Engineering, Novartis, Bayer, Sanofi Aventis, Allergan, and Bausch and Lomb.

\section{References}

1 Casswell AG, Kohen D, Bird AC: Retinal pigment epithelial detachments in the elderly: classification and outcome. Br J Ophthalmol 1985;69:397-403.

$\checkmark 2$ Yeo JH, Marcus S, Murphy RP: Retinal pigment epithelial tears. Patterns and prognosis. Ophthalmology 1988;95:8-13.

3 Pauleikhoff D, Löffert D, Spital G, Radermacher M, Dohrmann J, Lommatzsch A, Bird AC: Pigment epithelial detachment in the elderly. Clinical differentiation, natural course and pathogenetic implications. Graefes Arch Clin Exp Ophthalmol 2002;240:533-538.

$\checkmark 4$ Hoskin A, Bird AC, Sehmi K: Tears of detached retinal pigment epithelium. Br J Ophthalmol 1981;65:417-422.

5 Gelisken F, Inhoffen W, Partsch M, Schneider U, Kreissig I: Retinal pigment epithelial tear after photodynamic therapy for choroidal neovascularization. Am J Ophthalmol 2001; 131:518-520

6 Gass JD: Retinal pigment epithelial rip during krypton red laser photocoagulation. Am J Ophthalmol 1984;98:700-706.

7 Thompson JT: Retinal pigment epithelial tear after transpupillary thermotherapy for cho- roidal neovascularization. Am J Ophthalmol 2001;131:662-664.

-8 Singh RP, Sears JE: Retinal pigment epithelial tears after pegaptanib injection for exudative age-related macular degeneration. Am J Ophthalmol 2006;142:160-162.

\9 Shah CP, Hsu J, Garg SJ, Fischer DH, Kaiser R: Retinal pigment epithelial tear after intravitreal bevacizumab injection. Am J Ophthalmol 2006;142:1070-1072.

10 Carvounis PE, Kopel AC, Benz MS: Retinal pigment epithelium tears following ranibizumab for exudative age-related macular degeneration. Am J Ophthalmol 2007; 143:504-505.

-11 Saito M, Kano M, Itagaki K, Oguchi Y, Sekiryu T: Retinal pigment epithelium tear after intravitreal aflibercept injection. Clin Ophthalmol 2013;7:1287-1289.

12 Meyer CH, Mennel S, Schmidt JC, Kroll P: Acute retinal pigment epithelial tear following intravitreal bevacizumab (Avastin) injection for occult choroidal neovascularisation secondary to age related macular degeneration. Br J Ophthalmol 2006;90:1207-1208.

13 Chan CK, Abraham P, Meyer CH, Kokame GT, Kaiser PK, Rauser ME, Gross JG, Nuthi
AS, Lin SG, Daher NS: Optical coherence tomography-measured pigment epithelial detachment height as a predictor for retinal pigment epithelial tears associated with intravitreal bevacizumab injections. Retina 2010;30: 203-211.

14 Chang LK, Flaxel CJ, Lauer AK, Sarraf D: RPE tears after pegaptanib treatment in age-related macular degeneration. Retina 2007;27: 857-863.

15 Clemens CR, Wolf A, Alten F, Milojcic C, Eter $\mathrm{N}$ : Monthly treatment of ranibizumab in vascular pigment epithelium detachment due to age-related macular degeneration. ARVO 2015; Poster 2838 - C0066.

-16 Sarraf D, Chan C, Rahimy E, Abraham P: Prospective evaluation of the incidence and risk factors for the development of RPE tears after high- and low-dose ranibizumab therapy. Retina 2013;33:1551-1557.

$\checkmark 17$ Guber J, Praveen A, Saeed MU: Higher incidence of retinal pigment epithelium tears after ranibizumab in neovascular age-related macular degeneration with increasing pigment epithelium detachment height. Br J Ophthalmol 2013;97:1486-1487. 
18 Smith BT, Kraus CL, Apte RS: Retinal pigment epithelial tears in ranibizumab-treated eyes. Retina 2009;29:335-339.

19 Doguizi S, Ozdek S: Pigment epithelial tears associated with anti-VEGF therapy: incidence, long-term visual outcome, and relationship with pigment epithelial detachment in age-related macular degeneration. Retina 2014;34:1156-1162.

20 Chiang A, Chang LK, Yu F, Sarraf D: Predictors of anti-VEGF-associated retinal pigment epithelial tear using FA and OCT analysis. Retina 2008;28:1265-1269.

21 Wong LJ, Desai RU, Jain A, et al: Surveillance for potential adverse events associated with the use of intravitreal bevacizumab for retinal and choroidal vascular disease. Retina 2008; 28:1151-1158.

-22 Wolf A, Rüping J, Neubauer AS, Mayer W, Ulbig M, Haritoglou C, Holz FG, Eter N, Kampik A: Alterations of vascular pigment epithelium detachments associated with agerelated macular degeneration during upload with intravitreal ranibizumab. Retina 2013; 33:1843-1849.

23 Mennel S, Callizo J, Schmidt JC, Meyer CH: Acute retinal pigment epithelial tear in the untreated fellow eye following repeated bevacizumab (Avastin) injections. Acta Ophthalmol Scand 2007;85:689-691.

-24 Sarraf D, Reddy S, Chiang A, Yu F, Jain A: A new grading system for retinal pigment epithelial tears. Retina 2010;30:1039-1045.

25 von Rückmann A, Fitzke FW, Bird AC: Distribution of fundus autofluorescence with a scanning laser ophthalmoscope. Br J Ophthalmol 1995;79:407-412.

-26 Clemens CR, Alten F, Baumgart C, Heiduschka P, Eter N: Quantification of retinal pigment epithelium tear area in age-related macular degeneration. Retina 2014;34:24-31.

27 Caramoy A, Fauser S, Kirchhof B: Fundus autofluorescence and spectral domain optical coherence tomography findings suggesting tissue remodelling in retinal pigment epithelium tear. Br J Ophthalmol 2010;96:12111216.

-28 Bindewald A, Schmitz-Valckenberg S, Jorzik JJ, Dolar-Szczasny J, Sieber H, Keilhauer C, Weinberger AW, Dithmar S, Pauleikhoff D, Mansmann U, Wolf S, Holz FG: Classification of abnormal fundus autofluorescence patterns in the junctional zone of geographic atrophy in patients with age related macular degeneration. Br J Ophthalmol 2005;89:874878.

29 Nagiel A, Freund KB, Spaide RF, Munch IC, Larsen M, Sarraf D: Mechanism of retinal pigment epithelium tear formation following intravitreal anti-vascular endothelial growth factor therapy revealed by spectral-domain optical coherence tomography. Am J Ophthalmol 2013;156:981-988.

30 Chuang EL, Bird AC: The pathogenesis of tears of the retinal pigment epithelium. Am J Ophthalmol 1988;105:285-290.
31 Gass JDM: Pathogenesis of tears of the retinal pigment epithelium. Br J Ophthalmol 1984; 68:513-519.

32 Toth CA, Pasquale AC III, Graichen DF: Clinicopathologic correlation of spontaneous retinal pigment epithelial tears with choroidal neovascular membranes in age-related macular degeneration. Ophthalmology 1995;102: 272-277.

33 Lafaut BA, Aisenbrey S, Vanden Broecke C, Krott R, Jonescu-Cuypers CP, Reynders S, Bartz-Schmidt KU: Clinicopathological correlation of retinal pigment epithelial tears in exudative age-related macular degeneration: pretear, tear, and scarred tear. Br J Ophthalmol 2001;85:454-460.

34 Clemens CR, Bastian N, Alten F, Milojcic C, Heiduschka P, Eter N: Prediction of retinal pigment epithelial tear in serous vascularized pigment epithelium detachment. Acta Ophthalmol 2014;92:50-56.

35 Spaide RF: Enhanced depth imaging optical coherence tomography of retinal pigment epithelial detachment in age-related macular degeneration. Am J Ophthalmol 2009;147: 644-652.

36 Decker WL, Sanborn GE, Ridley M, Annesley WH Jr, Sorr EM: Retinal pigment epithelial tears. Ophthalmology 1983;90:507-512.

- 37 Chan CK, Meyer CH, Gross JG, Abraham P, Nuthi AS, Kokame GT, Lin SG, Rauser ME, Kaiser PK: Retinal pigment epithelial tears after intravitreal bevacizumab injection for neovascular age-related macular degeneration. Retina 2007;27:541-551.

38 Ie D, Yannuzzi LA, Spaide RF, Woodward KP, Singerman LJ, Blumenkranz MS: Microrips of the retinal pigment epithelium. Arch Ophthalmol 1992;110:1443-1449.

39 Sarraf D, Chan C, Rahimy E, Abraham P: Prospective evaluation of the incidence and risk factors for the development of RPE tears after high- and low-dose ranibizumab therapy. Retina 2013;33:1551-1557.

40 Leitritz M, Gelisken F, Inhoffen W, Voelker M, Ziemssen F: Can the risk of retinal pigment epithelium tears after bevacizumab treatment be predicted? An optical coherence tomography study. Eye 2008;22:1504-1507.

41 Clemens CR, Alten F, Eter N: Reading the signs: microrips as a prognostic sign for impending RPE tear development. Acta Ophthalmol 2015, Epub ahead of print.

42 Mukai R, Sato T, Kishi S: Precursor stage of retinal pigment epithelial tear in age-related macular degeneration. Acta Ophthalmol 2014;92:407-408.

43 Chan CK, Abraham P, Sarraf D, Nuthi AS, Lin SG, McCannel CA: Earlier therapeutic effects associated with high dose $(2.0 \mathrm{mg})$ ranibizumab for treatment of vascularized pigment epithelial detachments in age-related macular degeneration. Eye 2015;29:80-87.
44 Stewart MW, Rosenfeld PJ: Predicted biological activity of intravitreal VEGF trap. Br J Ophthalmol 2008;92:667-668.

45 Bertelmann T, Sekundo W, Wenner Y: Tear in the retinal pigment epithelium by intravitreal injection of aflibercept. Ophthalmologe 2014;111:775-777.

-46 Cunningham ET Jr, Feiner L, Chung C, Tuomi L, Ehrlich JS: Incidence of retinal pigment epithelial tears after intravitreal ranibizumab injection for neovascular age-related macular degeneration. Ophthalmology 2011; 118:2447-2452.

47 Coco RM, Sanabria MR, Hernandez AG, Fernández Muñoz M: Retinal pigment epithelium tears in age-related macular degeneration treated with antiangiogenic drugs: a controlled study with long follow-up. Ophthalmologica 2012;228:78-83.

48 Lesniak SP, Fine HF, Prenner JL, Roth DB: Long-term follow-up of spontaneous retinal pigment epithelium tears in age-related macular degeneration treated with anti-VEGF therapy. Eur J Ophthalmol 2011;21:73-76.

-49 Bartels S, Barrelmann A, Book B, Heimes B, Gutfleisch M, Spital G, Pauleikhoff D, Lommatzsch A: Tear in retinal pigment epithelium under anti-VEGF therapy for exudative age-related macular degeneration: function recovery under intensive therapy. Ophthalmologe 2014;111:460-464.

50 Asao K, Gomi F, Sawa M, Nishida K: Additional anti-vascular endothelial growth factor therapy for eyes with a retinal pigment epithelial tear after the initial therapy. Retina 2014; 34:512-518.

51 Mukai R, Sato T, Kishi S: Repair mechanism of retinal pigment epithelial tears in age-related macular degeneration. Retina 2015;35: 473-480.

52 Mendis R, Lois N: Fundus autofluorescence in patients with retinal pigment epithelial (RPE) tears: an in-vivo evaluation of RPE resurfacing. Graefes Arch Clin Exp Ophthalmol 2014;252:1059-1063.

53 Leonard DS, Zhang XG, Panozzo G, Sugino IK, Zarbin MA: Clinicopathological correlation of localized retinal pigment epithelium debridement. Invest Ophthalmol Vis Sci 1997;38:1094-1109.

54 Del Priore LV, Kuo YH, Tezel TH: Age related changes in human RPE cell density and apoptosis proportion in situ. Invest Ophthalmol Vis Sci 2002;43:3312-3318.

55 Caramoy A, Kirchhof B, Fauser S: Morphological versus functional photoreceptor viability of retinal pigment epithelium tears. Acta Ophthalmol 2012;90:328-329.

56 Stanzel BV, Liu Z, Somboonthanakij S, Wongsawad W, Brinken R, Eter N, Corneo B, Holz FG, Temple S, Stern JH, Blenkinsop TA: Human RPE stem cells grown into polarized RPE monolayers on a polyester matrix are maintained after grafting into rabbit subretinal space. Stem Cell Reports 2014;2:64-77. 